COHERE - Centre of Health Economics Research

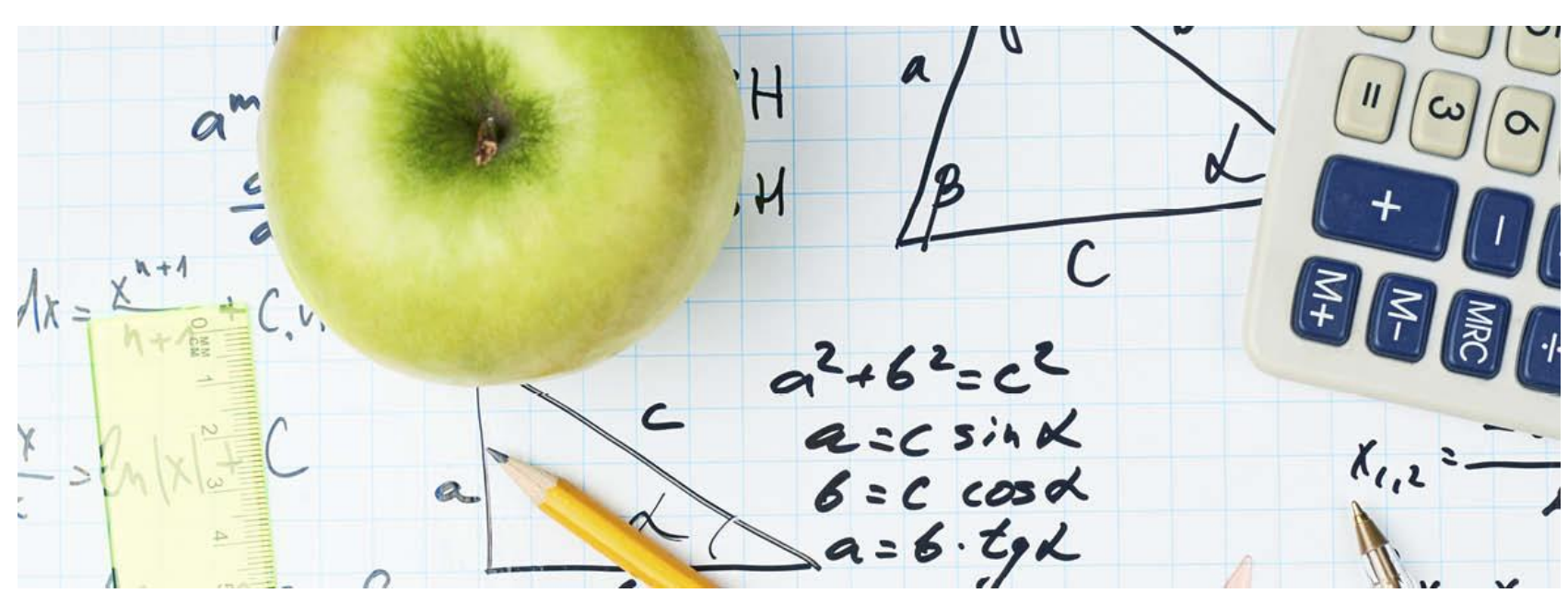

Dynamic Changes in Determinants of Inequalities in Health in Europe with Focus on Retired - with particular Regard to Retired Danes

\author{
By \\ Terkel Christiansen and Jørgen T. Lauridsen ${ }^{1}$ \\ COHERE, Department of Business and Economics, University of Southern Denmark
}

COHERE discussion paper No. 8/2016

FURTHER INFORMATION Department of Business and Economics Faculty of Business and Social Sciences University of Southern Denmark Campusvej 55 
E-mail tch@sam.sdu.dk and $\underline{\text { ttl@sam.sdu.dk }}$

${ }^{1}$ : Corresponding author 


\title{
Dynamic Changes in Determinants of Inequalities in Health in Europe with Focus on Retired - with particular Regard to retired Danes
}

\begin{abstract}
Earlier studies of health inequality across European countries have shown intriguing results, in particular with respect to retirement status as one of the determinants of health inequality. A priori, one would expect that inequality in health and income would be associated.
\end{abstract}

Theory suggests that health deteriorates with age, in particular for low income groups. Moreover, as income declines after retirement, elderly people tend to rank lower in the relative income ranking. Consequently, retirement status, and in particular early retirement due to health problems, is expected to contribute to inequalities in income-related inequalities in health.

The present paper contributes to previous knowledge by looking further into the contribution by retired Europeans to income-related inequalities in health and the development in this contribution over time. The study is based on data from the first and the fourth waves of the Survey of Health, Ageing and Retirement in Europe (SHARE), including individuals born in 1954 or earlier (wave 1) and 1960 or earlier (wave 2) from 10 European countries.

Income-related inequality in health is measured using the concentration index. A decomposition of the index into its determinants allows a calculation of the contribution of each determinant's separate contribution to inequality in health.

The results presented here indicate that retirement status contributes substantially to income-related inequality in health across European countries, and that the variation can be explained by income differences as well as health differences, depending on the country considered. Furthermore, it is indicated that the contribution from retirement status falls for certain countries due to improved socioeconomic status as well as improved health of the retired. 


\section{JEL Classifications}

114, J26

\section{Key Words}

Health inequality, retirement, SHARE data.

\section{Notes}

Earlier versions of this paper have been presented at the yearly meeting of the Nordic Health Economists' Study group in Tartu, August 16-17, 2007, and at the 2nd Biennial Conference of the American Society of Health Economists, Duke University, Durham, North Carolina, June 22-25, 2008. A preliminary version can be seen as a COHERE discussion paper by Christiansen et al. (2009). The present paper provides revision and update of earlier findings.

\section{Acknowledgement}

This paper uses data from SHARE wave 4 release 1.1.1, as of March 28th 2013 (DOI: 10.6103/SHARE.w4.111) or SHARE waves 1 and 2 release 2.6.0, as of November 29th 2013 (DOIs: 10.6103/SHARE.w1.260 and 10.6103/SHARE.w2.260) or SHARELIFE release 1.0.0, as of November 24th 2010 (DOI: 10.6103/SHARE.w3.100). The SHARE data collection has been primarily funded by the European Commission through the 5th Framework Programme (project QLK6-CT-2001-00360 in the thematic programme Quality of Life), through the 6th Framework Programme (projects SHARE-I3, RII-CT-2006-062193, COMPARE, CIT5- CT-2005-028857, and SHARELIFE, CIT4-CT-2006-028812) and through the 7th Framework Programme (SHARE-PREP, № 211909, SHARE-LEAP, N²27822 and SHARE M4, N²61982). Additional funding from the U.S. National Institute on Aging (U01 AG09740-13S2, P01 AG005842, P01 AG08291, P30 AG12815, R21 AG025169, Y1-AG-4553-01, IAG BSR06-11 and OGHA 04-064) and the German Ministry of Education and Research as well as from various national sources is gratefully acknowledged (see www.share-project.org for a full list of funding institutions).

We are grateful to Gawain Heckley, University of Lund, for his valuable comments to the paper at the Nordic Health Economists' Study Group meeting, Uppsala , August 2015. His comments have been included in the discussion. 


\section{Introduction}

Equality in health and health care is an important health policy objective in most European countries (van Doorslaer et al., 1993), and a long list of empirical studies have shown the existence of inequalities. Of particular interest has been the income-related inequality. Thus, income-related inequality in self-assessed health was reported by van Doorslaer et al. $(1993,1997,2004))$. The last study was based on the European Community Household Panel (ECHP), (EUROSTAT 1999) and showed unexpected results for Denmark. While Denmark was ranked at the top with respect to the level of self-assessed good health, it was ranked near the bottom with respect income-related distribution of health meaning a relatively high inequity (i.e., lowincome groups being least healthy when controlled for age and gender). Similar results for Denmark were found by Christiansen (1997). Specifically, these findings were unexpected because the income distribution in Denmark was among the most equal, and because inequality in income and health tends to be associated (van Doorslaer and Koolman, 2004). However, no significant association between income and health was found for Denmark. It was suggested that the relatively high income-related health inequality for Denmark was almost entirely due to a group of early retired with much poorer health who were strongly concentrated among the lower income groups. Thus, retirement might be seen as a way to withdraw early from the labour market due to health problems (van Doorslaer and Koolman, 2004). When using the same ECHP data from the $3^{\text {rd }}$ wave in 1996, and dividing the retired into three age groups $(-64$, 65-74 and 75-) we confirmed the existence of a relatively high income-related inequality in health for Denmark, compared to most West European countries (Lauridsen et al. 2008). We also found that retired of all three age groups were characterised by high income-inequality, in particular the two older age groups, and together they contributed by $67 \%$ to the income-related inequality in health ( $82 \%$ for $2^{\text {nd }}$ wave). In other words, if income had been equally distributed within each age group among retired, the measure of inequality in health would have been reduced by corresponding percentages (Christiansen et al. 2009).

In Denmark all pensioners above the age of 65 years have a right to universal, public pension in addition to any private pension (gradually increasing to 67 years from 2006). Since the studies referred to were conducted the relative income of pensioners have changed, and more pensioners have access to private pensions, either individually contracted or contracted as a part of an agreement of the labour market. Still, 
the relative income of Danes over 65 as percentage of the national mean income of the total population in the late 2000s was below the OECD average and below the average of nine other European countries to be included in the present study $\mathbf{7 4 . 3 \%}$ for Denmark as compared to an OECD average of $86 \%$. Among 33 OECD countries, Denmark ranked as number 32 with respect to relative income of pensioners (OECD 2013).

Access to international comparative data from European countries allows a comparative study of determinants of income-related inequalities in health and contribution from each factor. Of particular interest, due to the aforementioned earlier findings, are the contributions from retirement. These contributions will be further elaborated on in what follows.

\section{Purpose}

The aim of the present paper is to investigate the determinants of income-related inequalities in health and the contribution from these determinants to health-related inequality in health across European countries with particular focus on retirement as a determinant.

Due to earlier findings showing the Danish case to be an outlier with respect to contribution from retirement to health inequality, particular attention will be paid to Denmark and the development in over time here. 


\section{Data}

Data is from The Survey of Health, Ageing and Retirement in Europe (SHARE, wave 1 and 4, $2004-06$ and 2011-12, respectively) (SHARE-project, 2015). The SHARE target population of individuals in the surveys was defined as all individuals born in 1954 or earlier in Wave 1, or 1960 or earlier in Wave 4, "... who have their regular domicile in the respective SHARE country. A person is excluded if she or he is incarcerated, hospitalized or out of the country during the entire survey period, unable to speak the country's language(s) or has moved to an unknown address. In addition, current partners living in the household are interviewed regardless of their age" (Börsh-Supan et al., 2013). As defined, people living in nursing homes were included in the target population. We have used SHARE data because it allowed comparisons between a range of countries and over time. A disadvantage is that the data only includes individuals above 50 years, but this still allows a focus on retired.

Ten countries, which entered both waves, were selected for the study: Austria, Germany, Sweden, Netherlands, Spain, Italy, France, Denmark, Switzerland and Belgium. Tables 1 and 2 show means of variables and numbers of observations for these ten countries for $1^{\text {st }}$ and $4^{\text {th }}$ wave respectively.

The variables include health status and demographic and socio-economic variables. Predicted health is based on a question about self-assessed health with five response categories. For half of wave 1 (selected at random) and wave 4, the categories were coded as "very bad", "bad", "fair", "good" and "very good", while the coding for the remaining half of the wave 1 respondents the coding "poor", "fair", "good", "very good" and "excellent" was used. As an operationally simple adjustment for the upward shift in the latter version, a dummy variable was coded for the respondents asked this version. When health is measured by such a self-assessed health $(\mathrm{SAH})$ scale with ordered categories, an ordinal scale is obtained. This can be transformed to a cardinal scale by using a mapping method to scale the thresholds, based on already known scores from another survey which has included both the SAH measure and an instrument allowing a cardinal measure. Our approach is based the HUI-3 (Health Utility Index, version 3) instrument that was included along with the SAH measure in a previous Canadian survey, National Population Survey (NPS) (van 
Doorslaer et al., 1997; van Doorslaer and Jones, 2003). Throughout, we use the terms "predicted health" and "health" synonymously.

Table 1. Means of variables, Wave 1

\begin{tabular}{|c|c|c|c|c|c|c|c|c|c|c|}
\hline & Austria & Germany & Sweden & Netherlands & Spain & Italy & France & Denmark & Switzerland & Belgium \\
\hline Health (pred.) & 0.826 & 0.803 & 0.862 & 0.852 & 0.777 & 0.789 & 0.811 & 0.849 & 0.880 & 0.839 \\
\hline Log(Income) & 10.108 & 10.223 & 12.643 & 10.297 & 9.422 & 9.744 & 10.088 & 12.483 & 11.031 & 9.959 \\
\hline Male 50-59 (ref) & 0.158 & 0.173 & 0.169 & 0.219 & 0.14 & 0.158 & 0.224 & 0.237 & 0.202 & 0.206 \\
\hline Male 60-69 & 0.170 & 0.187 & 0.155 & 0.153 & 0.131 & 0.164 & 0.116 & 0.135 & 0.145 & 0.130 \\
\hline Male 70- & 0.108 & 0.118 & 0.154 & 0.121 & 0.162 & 0.138 & 0.132 & 0.123 & 0.148 & 0.147 \\
\hline Female 50-59 & 0.160 & 0.181 & 0.195 & 0.224 & 0.185 & 0.184 & 0.198 & 0.193 & 0.192 & 0.196 \\
\hline Female 60-69 & 0.207 & 0.189 & 0.170 & 0.147 & 0.161 & 0.204 & 0.136 & 0.136 & 0.145 & 0.146 \\
\hline Female 70- & 0.197 & 0.152 & 0.157 & 0.136 & 0.221 & 0.152 & 0.194 & 0.176 & 0.168 & 0.175 \\
\hline Employed (ref) & 0.804 & 0.763 & 0.888 & 0.619 & 0.510 & 0.676 & 0.781 & 0.862 & 0.76 & 0.706 \\
\hline Self-employed & 0.035 & 0.050 & 0.052 & 0.045 & 0.058 & 0.068 & 0.042 & 0.043 & 0.101 & 0.040 \\
\hline Unemployed & 0.023 & 0.054 & 0.022 & 0.021 & 0.032 & 0.014 & 0.036 & 0.046 & 0.014 & 0.047 \\
\hline Housework & 0.124 & 0.107 & 0.010 & 0.236 & 0.357 & 0.231 & 0.115 & 0.018 & 0.093 & 0.162 \\
\hline Disabled & 0.014 & 0.026 & 0.028 & 0.079 & 0.043 & 0.011 & 0.026 & 0.031 & 0.032 & 0.045 \\
\hline Not retired (ref) & 0.345 & 0.489 & 0.471 & 0.653 & 0.634 & 0.447 & 0.485 & 0.489 & 0.543 & 0.508 \\
\hline Retired -64 & 0.244 & 0.109 & 0.082 & 0.064 & 0.062 & 0.197 & 0.120 & 0.128 & 0.049 & 0.123 \\
\hline Retired 65-74 & 0.248 & 0.263 & 0.255 & 0.170 & 0.169 & 0.239 & 0.220 & 0.197 & 0.215 & 0.211 \\
\hline Retired 75- & 0.163 & 0.139 & 0.192 & 0.113 & 0.135 & 0.117 & 0.175 & 0.186 & 0.193 & 0.158 \\
\hline Single & 0.395 & 0.235 & 0.223 & 0.179 & 0.253 & 0.216 & 0.317 & 0.354 & 0.290 & 0.258 \\
\hline Short education (ref) & 0.324 & 0.178 & 0.524 & 0.566 & 0.854 & 0.773 & 0.529 & 0.251 & 0.525 & 0.509 \\
\hline Medium education & 0.484 & 0.568 & 0.262 & 0.234 & 0.074 & 0.174 & 0.281 & 0.435 & 0.387 & 0.259 \\
\hline Long education & 0.192 & 0.254 & 0.214 & 0.200 & 0.072 & 0.053 & 0.190 & 0.314 & 0.088 & 0.232 \\
\hline Foreign & 0.017 & 0.024 & 0.024 & 0.015 & 0.014 & 0 & 0.055 & 0.013 & 0.077 & 0.037 \\
\hline SAH version 2 & 0.510 & 0.500 & 0.507 & 0.498 & 0.501 & 0.504 & 0.501 & 0.494 & 0.503 & 0.486 \\
\hline $\mathrm{N}$ & 1576 & 2977 & 2992 & 2873 & 2322 & 2493 & 3041 & 1689 & 966 & 3773 \\
\hline
\end{tabular}

Rather than OLS we use interval regression as defined below under Methodology due to the nature of the health variable with five groups. In the first wave the predicted value of health ranges from 0.777 in Spain and 0.789 in Italy, followed by Germany and France, to 0.862 in Sweden and 0.88 in Switzerland. In the fourth wave predicted health status was on average 0.05 points lower for each county, except for Denmark with no substantial difference between the waves. The relatively low values for Spain and Italy were expected since these countries have a relatively high population share of elder, and health tends to decline by increasing age. 
Table 2. Means of variables, Wave 4

\begin{tabular}{|c|c|c|c|c|c|c|c|c|c|c|}
\hline & Austria & Germany & Sweden & Netherlands & Spain & Italy & France & Denmark & Switzerland & Belgium \\
\hline Health (pred.) & 0.794 & 0.730 & 0.787 & 0.798 & 0.699 & 0.734 & 0.742 & 0.842 & 0.841 & 0.783 \\
\hline Log(Income) & 9.882 & 9.853 & 10.206 & 10.178 & 9.154 & 9.546 & 9.952 & 10.344 & 10.964 & 10.267 \\
\hline Male 50-59 (ref) & 0.153 & 0.065 & 0.050 & 0.122 & 0.134 & 0.118 & 0.171 & 0.190 & 0.170 & 0.181 \\
\hline Male 60-69 & 0.147 & 0.174 & 0.184 & 0.185 & 0.134 & 0.158 & 0.142 & 0.154 & 0.159 & 0.148 \\
\hline Male 70- & 0.145 & 0.231 & 0.227 & 0.146 & 0.202 & 0.192 & 0.144 & 0.131 & 0.146 & 0.140 \\
\hline Female 50-59 & 0.166 & 0.112 & 0.068 & 0.162 & 0.150 & 0.141 & 0.173 & 0.197 & 0.188 & 0.200 \\
\hline Female 60-69 & 0.193 & 0.203 & 0.232 & 0.214 & 0.153 & 0.195 & 0.167 & 0.164 & 0.169 & 0.154 \\
\hline Female 70- & 0.196 & 0.215 & 0.239 & 0.171 & 0.227 & 0.196 & 0.203 & 0.164 & 0.168 & 0.177 \\
\hline Employed (ref) & 0.813 & 0.833 & 0.932 & 0.723 & 0.558 & 0.692 & 0.84 & 0.868 & 0.789 & 0.737 \\
\hline Self-employed & 0.053 & 0.040 & 0.035 & 0.043 & 0.053 & 0.053 & 0.039 & 0.053 & 0.090 & 0.047 \\
\hline Unemployed & 0.024 & 0.027 & 0.011 & 0.017 & 0.060 & 0.017 & 0.029 & 0.024 & 0.015 & 0.048 \\
\hline Housework & 0.100 & 0.078 & 0.005 & 0.160 & 0.286 & 0.212 & 0.060 & 0.006 & 0.085 & 0.118 \\
\hline Disabled & 0.010 & 0.022 & 0.017 & 0.057 & 0.043 & 0.026 & 0.032 & 0.049 & 0.021 & 0.050 \\
\hline Not retired (ref) & 0.370 & 0.353 & 0.307 & 0.542 & 0.593 & 0.46 & 0.408 & 0.529 & 0.549 & 0.516 \\
\hline Retired -64 & 0.205 & 0.082 & 0.044 & 0.060 & 0.043 & 0.113 & 0.149 & 0.053 & 0.037 & 0.113 \\
\hline Retired $65-74$ & 0.271 & 0.339 & 0.374 & 0.240 & 0.171 & 0.259 & 0.224 & 0.228 & 0.242 & 0.199 \\
\hline Retired 75- & 0.154 & 0.226 & 0.275 & 0.158 & 0.193 & 0.168 & 0.219 & 0.190 & 0.172 & 0.172 \\
\hline Single & 0.349 & 0.202 & 0.226 & 0.184 & 0.191 & 0.179 & 0.328 & 0.279 & 0.262 & 0.303 \\
\hline Short education (ref) & 0.247 & 0.126 & 0.453 & 0.465 & 0.81 & 0.698 & 0.448 & 0.176 & 0.195 & 0.426 \\
\hline Medium education & 0.499 & 0.567 & 0.281 & 0.267 & 0.099 & 0.235 & 0.345 & 0.397 & 0.645 & 0.268 \\
\hline Long education & 0.254 & 0.307 & 0.266 & 0.268 & 0.091 & 0.067 & 0.207 & 0.427 & 0.160 & 0.306 \\
\hline Foreign & 0.028 & 0.000 & 0.028 & 0.012 & 0.019 & 0.001 & 0.032 & 0.018 & 0.083 & 0.038 \\
\hline $\mathrm{N}$ & 5047 & 1469 & 1846 & 2562 & 3296 & 3393 & 5518 & 2165 & 3580 & 4980 \\
\hline
\end{tabular}

Focus is on retired, and we use economic status, disability and single as possible confounders (van Doorslaer and Koolman, 2000). Our reference group for retired is wage earners.

Demographic structure of a country is shown by combined age- and gender variables. Reference group is males less than or equal to 59 years old. The tables show some difference in the age- and gender composition between countries and over time from $1^{\text {st }}$ to $4^{\text {th }}$ wave. Thus, in wave 1 , the group with females in the age group 50-69 years was relatively high in The Netherlands with more than $20 \%$ while females 60 69 years made up a relatively high share with about $20 \%$ of the sample in Austria and Italy, closely followed by Germany and Sweden. Females above 70 years made up a relatively high share in Spain with $22 \%$, followed by Austria and France with close to $20 \%$. Men between 60 and 69 years made up a relatively high share in Germany and Austria, while men above 70 years made up a relatively high share in Spain and Sweden. In wave 4 females 50-59 years old made up a relatively high share in Belgium, followed by Switzerland and Denmark (20\%). Females in the age group 60-69 years make up more than $20 \%$ in Sweden, The Netherlands and Germany while the oldest age group make up more than $20 \%$ in Sweden, Spain and Germany. For men above 70 years the relatively largest shares were found in Germany, Sweden and Spain with more than $20 \%$. These differences may be a reflection of the age composition and different response 
rates as well. Disabled made up between $1 \%$ and $6 \%$ in wave 1 and between 1 and $5 \%$ in wave 4 . Hence, disabled made only a small share of the samples.

While the retired who are less than 65 years as a share of the total sample in wave 1 was particularly low in Sweden, The Netherlands, Spain and Switzerland with between $5 \%$ and $8 \%$ of the samples, the share varied between $12 \%$ and $13 \%$ in France, Denmark and Belgium, and in Italy the share reached 20\%. Austria appeared to be an outlier with $24 \%$. Thus, retired below 65 years as percentage of the sample varied substantially between the countries. In wave 4, the shares have decreased in most countries with a range between 4\% in Switzerland and Sweden and 15\% in France and 21\% in Austria. Obviously, this reflects both the pattern of retirement, other means of being supported when not active on the labour market (doing housework, being unemployed or disabled), the age composition and possible differences in response rates. The shares above 75 years varied between $11 \%$ in Netherlands to $19 \%$ in Sweden and Switzerland in wave 1 and between $15 \%$ in Austria to $27 \%$ in Sweden in wave 4 . Clearly, the age composition of retired varied substantially between these countries with varying degrees of ageing populations.

The percentage possessing a secondary or higher education also varied quite substantially between countries from $15 \%$ in Spain to $82 \%$ in Germany (wave 1). Obviously, this reflects past education policy and possibilities. In wave 4, the educational level appeared higher and the range more narrow. Table 2 shows corresponding data from wave 4.

Income was measured as disposable household income, that is, monetary income in purchasing power adjusted Euro after income tax. No correction was made for indirect social transfers like subsidies to medical care and medicine or rental of housing, services in kind or calculated value of own dwelling in accordance with the approach by van Doorslaer et al. (2004). Household income was adjusted for the household composition by using OECD's modified equivalence scale (with 1 for $1^{\text {st }}$ adult; 0.5 for the $2^{\text {nd }}$ and children above 14 years; 0.3 for each child below 14 years) (OECD Social Policy Division). Being measured in purchasing power adjusted euro, income data are comparable across countries. Income is log transformed prior to the analysis; Tables 1 and 2 show the means of log to income. 
The reference group for the categorical variables is employed males with short education who are 50-59 year old, have short educations, live in households with more than one person and who are EU citizens. The very different rates of 'house worker' as employment status from less than $2 \%$ in Denmark to $36 \%$ in Spain (wave 1) should be expected as it reflects differences in female labour force participation.

The percentage possessing a secondary or higher education also varied quite substantially between countries from $14 \%$ in Spain to $81 \%$ in Germany in wave 1, but with a smaller range in wave 4 . Obviously, these figures reflect variations in educational policy and opportunities.

\section{Hypotheses}

One factor that may explain differences in the sizes of the concentration indices is the demographic composition of the samples. Thus, when controlled for age and gender, the differences in inequality in health between countries may change.

Status as retired from the labour market can be expected to be a factor that contributes to income-related inequality because retired typically have a lower income compared to active on the labour market, and because health status for retired in general is worse than for active, either because of age-related deterioration of health or because of ill-health as reason for retirement. To investigate how status as retired from the labour market contributes to income-related inequality in health, it is necessary to distinguish between the three factors that contribute to the measure, namely their share of the total population, their health status and distribution of income among retired. Additionally, it can be assumed that health and income are different for different age groups among the retired, both decreasing by increasing age.

This leads to the following hypotheses about retired in Denmark compared to retired in other countries in the sample as explanations for the relatively high Danish inequality in income-related health status:

1. The share of the population being retired is greater in Denmark compared to other countries (implying that they contribute more to the over-all inequality due to a higher share) 
2a. Retired have relatively worse health in Denmark, as compared to retired in other countries.

$2 \mathrm{~b}$. The relative health status of retired decreases by increasing age to a greater extent than in other countries.

3a. Retired in Denmark have lower relative incomes, defined as income ratio between retired and nonretired, as compared to other EU-countries.

3b. The relative income of retired decreases by increasing age to a greater extent than in other EUcountries.

The Danish case is characterised by an increasing use of private pensions since the early 1990s. These pensions are related to the labour market as a supplement to public financed universal age pension, and this may have resulted in an income inequality between younger and older age pensioners who have not been included in labour market pension schemes (Det $\varnothing$ konomiske Råd, 2008). The youngest among the retired (below 65 years) can be expected to be a mixed group of retired due to health and other reasons, and consequently no specific hypotheses are formulated for those. When distinguishing between pensioners 65-74 years and 75+ years it would be natural to expect a different contribution from these two groups to income-related inequality in health and that this difference has changed over time. Thus, private pensions can be expected to affect income more among the middle age groups compared to the oldest group where many have only have had a short time - if any - to save for their pension. This leads to the hypothesis:

4. The middle age group among pensioners contributes less to income-related inequality in health over time.

\section{Methods}

Similar to previous studies initiated by van Doorslaer et al. (1993) we use the concentration index as our measure of relative socioeconomic inequality in self-assessed health. A concentration curve $L(s)$ plots the 
cumulative proportion of the population (ranked by socioeconomic status (SES), beginning with lowest SES) against the cumulative proportion of health. If $L(s)$ coincides with the diagonal everyone is equally off. However, if $L(s)$ lies below the diagonal, then inequality in health exists and favors those with high SES. The further $L(s)$ lies from the diagonal, the greater the degree of inequality. The concentration index, $C$, is defined as twice the area between $L(s)$ and the diagonal and takes a value of 0 when everyone is equally of regardless of SES. The minimum and maximum values of $C$ are -1 and +1 , respectively; these occur in the (hypothetical) situation where health is concentrated in the hand of the least disadvantaged and the most disadvantaged person, respectively. Thus, the larger negative value of $C$, the more ill-health concentrates among low SES groups. A computational formula for $C$, which allows for application of sample weights was given by Kakwani et al. (1997) as $C=\frac{2}{N \mu} \sum_{i=1}^{N} w_{i} y_{i} R_{i}-1$, where $\mu=\frac{1}{N} \sum_{i=1}^{N} w_{i} y_{i}$ is the weighted mean of (predicted) health, $N$ the sample size, $y_{i}$ (predicted) health, $w_{i}$ the sample weight of the individual (which sums to $N$ ) and $R_{i}$ the fractional rank defined according to Kakwani et al. as $R_{i}=\frac{1}{N} \sum_{j=1}^{i-1} w_{j}+\frac{w_{i}}{2}$, i.e. the weighted cumulative proportion of the population up to the midpoint of each individual weight. Following the same authors, $C$ can be conveniently computed as the weighted covariance of $y_{i}$ and $R_{i}$, i.e. $C=\frac{2}{\mu} \operatorname{cov}_{w}\left(y_{i}, R_{i}\right)=\frac{2}{N \mu} \sum_{i=1}^{N} w_{i}\left(y_{i}-\mu\right)\left(R_{i}-\frac{1}{2}\right)$.

A straightforward way of decomposing the predicted degree of inequality into the contributions of explanatory factors was proposed by Wagstaff et al. (2003). Adapting their approach to the present case, where an interval regression specification (Jones, 2000) links health to the determinants, leads to a decomposition of the concentration index of predicted health as $\hat{C}=\sum_{k} \frac{\beta_{k} \bar{x}_{k}}{\hat{\mu}} C_{k}$, where $\hat{\mu}$ is the mean of predicted health, $\bar{x}_{k}$ the mean of the determinant $x_{k}$, and $C_{k}$ the concentration index of $x_{k}$ (defined analogously to $C$ ). 
In order to assess sampling variability and to obtain standard errors for the estimated quantities, where in particular the concentration indices and the contributions, i.e. the $\frac{\beta_{k} \bar{x}_{k}}{\hat{\mu}} C_{k}$ parts, cause troubles, we apply a "bootstrap" procedure (Efron and Tibshirani, 1993; Deaton, 1997) in a five-step manner much similar to van Doorslaer and Koolman, (2004): First, sample size is inflated to allow for differences in sampling probability by dividing the sampling weights with the smallest weight and rounding to nearest integer. Second, from this expanded sample a random sub-sample of the size of the original sample is drawn with replacement. Third, the entire set of calculations as specified above are performed on this sample. Fourth, this whole process is repeated 1,000 times, each leading to replicate estimates. Fifth, using the obtained 1,000 replicates, standard deviations and $t$ statistics can be computed. As focus is not on the magnitudes of the effects of the determinants on health, we report regression coefficients rather than marginal effects.

\section{Results}

Tables 3 and 4 show the estimated interval regression coefficients from wave 1 and 4 respectively. Dependent variable is the HUl-scaled self-assessed health. In both waves, income is positively associated with health status in all cases with a statistical significant estimate. A similar observation is made for secondary and tertiary education as compared to elementary education, while status as house worker, disabled, retired or being a non-EU citizen show negative associations.

With few exceptions in wave 1 , in particular for Denmark, most countries have coefficients of the demographic age-gender interaction variables that are significantly negative implying lower health (utility) as compared to the reference group (made up of males 50-59 years). In general, males and females above 70 years report lower health than this reference group. In wave 4, the results are more scattered, except that those over 70 years have lower health in most countries. For Sweden, there are no significant results, and for Denmark only two categories are vaguely significant with opposite signs.

\section{Table 3. Regression coefficients, Wave 1}




\begin{tabular}{|c|c|c|c|c|c|c|c|c|c|c|}
\hline & Austria & Germany & Sweden & Netherl. & Spain & Italy & France & Denmark & Switzerl. & Belgium \\
\hline Log(Income) & -0.005 & $0.007^{* * *}$ & $0.018^{* * *}$ & $0.006^{* *}$ & 0.003 & $0.013^{* * *}$ & $0.014^{* * *}$ & $0.022^{* * * *}$ & $0.009^{* * *}$ & 0.003 \\
\hline Male 60-69 & $0.025^{*}$ & $-0.030 * * *$ & 0.007 & -0.006 & -0.012 & -0.007 & $-0.024^{* * *}$ & $0.048 * * *$ & 0.011 & -0.005 \\
\hline Male 70- & 0.007 & $-0.049 * * *$ & $-0.022^{* *}$ & -0.016 & $-0.040 * *$ & $-0.055^{* * * *}$ & $-0.061 * * *$ & $0.038^{*}$ & -0.015 & $-0.024 * *$ \\
\hline Female 50-59 & $0.020^{*}$ & -0.004 & $-0.018^{* * *}$ & -0.001 & -0.010 & -0.008 & -0.004 & 0.004 & -0.002 & 0.002 \\
\hline Female 60-69 & 0.025 & -0.015 & 0.007 & 0.005 & $-0.053^{* * *}$ & $-0.025^{* *}$ & $-0.022^{* *}$ & $0.051^{* * *}$ & $-0.025^{*}$ & $-0.014^{*}$ \\
\hline Female 70- & -0.021 & $-0.055 * * *$ & $-0.027^{* *}$ & $-0.018^{*}$ & $-0.117 * * *$ & $-0.094 * * *$ & $-0.047 * * *$ & $0.047^{* *}$ & $-0.027^{*}$ & $-0.042^{* * *}$ \\
\hline Self-employed & -0.022 & $0.021^{* * * *}$ & 0.007 & 0.005 & -0.006 & 0.007 & -0.009 & -0.006 & 0.003 & -0.006 \\
\hline Unemployed & $-0.060 * * *$ & $-0.041 * * *$ & $-0.025^{* *}$ & $-0.026^{* *}$ & $-0.037^{*}$ & -0.032 & 0.005 & $-0.054 * * *$ & -0.015 & $-0.029 * * *$ \\
\hline Housework & $-0.032 * *$ & $-0.040 * * *$ & -0.026 & $-0.021 * * *$ & $-0.036^{* * *}$ & $-0.039 * * *$ & -0.016 & -0.011 & -0.010 & $-0.023^{* * *}$ \\
\hline Disabled & $-0.294 * * *$ & $-0.247 * * *$ & $-0.148^{* * *}$ & $-0.183^{* * *}$ & $-0.191^{* * *}$ & $-0.250 * * *$ & $-0.211^{* * *}$ & $-0.279 * * *$ & $-0.208^{* * * *}$ & $-0.211^{* * * *}$ \\
\hline Retired -64 & $-0.053^{* * *}$ & $-0.037 * * *$ & $-0.111 * * *$ & -0.004 & $-0.075^{* * *}$ & $-0.031 * * *$ & $-0.021 * *$ & $-0.100 * * *$ & -0.017 & $-0.020 * * *$ \\
\hline Retired $65-74$ & $-0.055^{* * *}$ & $-0.035 * * *$ & $-0.023^{* * *}$ & $-0.031 * * *$ & $-0.043^{* * *}$ & $-0.036 * * *$ & -0.017 & $-0.064 * * *$ & $-0.022^{*}$ & $-0.028^{* * *}$ \\
\hline Retired 75- & $-0.082^{* * *}$ & $-0.092^{* * *}$ & $-0.045^{* * *}$ & $-0.055^{* * *}$ & $-0.079 * * *$ & $-0.056 * * *$ & $-0.068 * * *$ & $-0.095 * * *$ & -0.024 & $-0.050 * * *$ \\
\hline Single & -0.001 & -0.008 & 0.003 & $-0.011 *$ & 0.010 & -0.005 & 0.001 & 0.008 & $0.013^{*}$ & -0.008 \\
\hline Medium education & $0.049 * * *$ & $0.038^{* * * *}$ & $0.014^{* * *}$ & $0.015^{* * *}$ & $0.037^{* * *}$ & $0.017^{* *}$ & $0.019^{* * *}$ & $0.029^{* * *}$ & 0.010 & $0.022^{* * *}$ \\
\hline Long education & $0.053^{* * *}$ & $0.055^{* * *}$ & $0.033^{* * *}$ & $0.021^{* * *}$ & $0.032^{* *}$ & $0.038^{* * *}$ & $0.050 * * *$ & $0.040^{* * *}$ & $0.030 * * *$ & $0.035 * * *$ \\
\hline Foreign & -0.018 & -0.028 & $-0.038^{* *}$ & $-0.080^{* * *}$ & 0.012 & NA & $-0.056 * * *$ & $-0.075^{*}$ & -0.011 & -0.022 \\
\hline SAH version 2 & $0.081^{* * *}$ & $0.102^{* * *}$ & $0.038^{* * *}$ & $0.079 * * *$ & $0.125^{* * *}$ & $0.115^{* * *}$ & $0.119 * * *$ & $0.059 * * *$ & $0.073^{* * *}$ & $0.085 * * *$ \\
\hline
\end{tabular}

Note. Significance indicated with ${ }^{*}(10 \%),{ }^{* *}(5 \%)$, and ${ }^{* * *}(1 \%)$

Not surprisingly, disabled consistently show a negative and substantial association with health. The three retired groups come up with consistently negative signs of the regression coefficients. Generally, the absolute values of these are larger for the older age groups, but there are exceptions. The reference group for these is non-retired above 50 years.

Table 4. Regression coefficients, Wave 4

\begin{tabular}{|c|c|c|c|c|c|c|c|c|c|c|}
\hline & Austria & Germany & Sweden & Netherl. & Spain & Italy & France & Denmark & Switzerl. & Belgium \\
\hline Log(Income) & 0.004 & 0.012 & $0.024 * * *$ & 0.006 & 0.003 & 0.001 & $0.019 * * *$ & $0.017^{* * *}$ & $0.008^{* * * *}$ & $0.008 * * *$ \\
\hline Male 60-69 & 0.013 & 0.027 & 0.012 & -0.006 & $-0.044 * * *$ & $-0.036 * * *$ & -0.003 & 0.006 & $-0.013^{*}$ & -0.002 \\
\hline Male 70- & -0.012 & 0.030 & -0.001 & -0.023 & $-0.084 * * *$ & $-0.065 * * *$ & $-0.041^{* * * *}$ & $-0.031^{*}$ & $-0.016^{*}$ & $-0.028^{* * *}$ \\
\hline Female 50-59 & $0.023^{* * *}$ & 0.047 & -0.018 & $-0.018^{* *}$ & $-0.027^{* * *}$ & 0.001 & -0.004 & -0.002 & -0.003 & $-0.011^{*}$ \\
\hline Female 60-69 & $0.047^{* * * *}$ & 0.037 & 0.008 & -0.006 & $-0.056 * * *$ & $-0.043^{* * *}$ & -0.003 & $0.024^{*}$ & -0.008 & -0.011 \\
\hline Female 70- & -0.006 & 9,933 & -0.011 & $-0.036^{* *}$ & $-0.148 * * *$ & $-0.129 * * *$ & $-0.040 * * *$ & -0.022 & $-0.021^{* *}$ & $-0.056 * * *$ \\
\hline Self-employed & 0.001 & 0.010 & 0.006 & 0.006 & -0.005 & 0.011 & -0.012 & -0.014 & 0.001 & $0.018^{* *}$ \\
\hline Unemployed & $-0.071^{* * *}$ & -0.084 & $-0.110^{* * *}$ & $-0.040^{*}$ & $-0.027^{* *}$ & $-0.068 * *$ & -0.016 & $-0.103^{* * *}$ & $-0.085^{* * *}$ & $-0.051^{* * *}$ \\
\hline Housework & $-0.074 * * *$ & -0.013 & -0.061 & $-0.028 * * *$ & $-0.066 * * *$ & $-0.052^{* * *}$ & $-0.034 * * *$ & $0.035 * *$ & -0.010 & $-0.035^{* * *}$ \\
\hline Disabled & $-0.277 * * *$ & -0.225 & $-0.366 * * *$ & $-0.202^{* * *}$ & $-0.299 * * *$ & $-0.310 * * *$ & $-0.283^{* * *}$ & $-0.222^{* * *}$ & $-0.318^{* * * *}$ & $-0.245^{* * *}$ \\
\hline Retired -64 & $-0.080 * * *$ & -0.016 & $-0.139 * * *$ & -0.006 & $-0.100 * * *$ & -0.007 & $-0.025 * * *$ & $-0.049 * * *$ & -0.006 & $-0.023^{* * *}$ \\
\hline Retired 65-74 & $-0.071 * * *$ & -0.033 & $-0.055^{* * *}$ & $-0.038 * * *$ & $-0.060 * * *$ & $-0.026^{* *}$ & $-0.040 * * *$ & $-0.019 *$ & -0.009 & $-0.031 * * *$ \\
\hline Retired 75- & $-0.089 * * *$ & -0.128 & $-0.099 * * *$ & $-0.050 * * *$ & $-0.083^{* * *}$ & $-0.095 * * *$ & $-0.089 * * *$ & $-0.045^{* *}$ & $-0.049 * * *$ & $-0.057^{* * *}$ \\
\hline Single & $-0.024 * * *$ & -0.007 & -0.013 & $-0.023 * *$ & 0.012 & $-0.027 * * *$ & -0.008 & $-0.015^{*}$ & -0.003 & -0.006 \\
\hline Medium education & $0.042^{* * * *}$ & 0.042 & $0.018^{*}$ & $0.026 * * *$ & $0.052^{* * * *}$ & $0.032^{* * *}$ & $0.035 * * *$ & $0.024 * *$ & $0.030 * * *$ & $0.030 * * *$ \\
\hline Long education & $0.065^{* * *}$ & 0.086 & $0.034^{* * * *}$ & $0.028 * * *$ & $0.060^{* * * *}$ & $0.057^{* * *}$ & $0.065^{* * *}$ & $0.047^{* * *}$ & $0.052^{* * *}$ & $0.052^{* * *}$ \\
\hline Foreign & $-0.013^{*}$ & -0.011 & 0.004 & 0.001 & 0.001 & -0.006 & -0.004 & -0.001 & -0.003 & $0.008^{*}$ \\
\hline
\end{tabular}

Note. Significance indicated with * $(10 \%),{ }^{* *}(5 \%)$, and $* * *(1 \%)$

Tables 5 and 6 show, for waves 1 and 4 respectively, the concentration indices for the dependent variable (predicted health, C) as well as independent variables. To measure the amount of inequality which cannot be ascribed to age or gender ('avoidable inequalities'), we first calculated the contribution to $C$ by age and gender, $\mathrm{C}^{*}$. The difference between $\mathrm{C}$ and $\mathrm{C}^{*}$ is the age- and gender standardized concentration index for 
health, $\mathrm{I}^{*}=\mathrm{C}-\mathrm{C}^{*} . \mathrm{I}^{*}$ was calculated in both absolute value as well as percentage of $\mathrm{C}$. Furthermore, concentration indices for the explanatory variables are shown.

Except for Austria, the concentration indices for predicted health, C, vary between 0.012 in Switzerland and 0.025 in Denmark. Austria seems to be an outlier for wave 1. Turning to wave 4, the index varies between 0.016 for Austria and Switzerland and 0.24 for Denmark. The variation in age and gender contributions to inequality is considerable as shown by the concentration index for 'avoidable inequality', $I^{*}$, which, in percentage of C, varies for wave 1 between $44 \%$ in Spain and 128\% in Denmark, where the age and gender contribution (i.e. $\mathrm{C}^{*}$ ) appears to be negative. In wave 4 , this range is narrowed to between $58 \%$ in Spain and $99,37 \%$ in Germany. Hence, while it is the case in all countries that predicted health is unequally distributed in favour if higher incomes, there are quite substantial differences.

Table 5. Concentration indices, Wave 1

\begin{tabular}{|c|c|c|c|c|c|c|c|c|c|c|}
\hline & Austria & Germany & Sweden & Netherl. & Spain & Italy & France & Denmark & Switzerl. & Belgium \\
\hline Health (pred.) & 0.005 & 0.023 & 0.019 & 0.013 & 0.016 & 0.020 & 0.022 & 0.025 & 0.012 & 0.015 \\
\hline $\mathrm{C}^{*}$ & 0.001 & 0.005 & 0.002 & 0.001 & 0.009 & 0.005 & 0.004 & -0.007 & 0.003 & 0.004 \\
\hline$I^{*}$ & 0.004 & 0.018 & 0.016 & 0.012 & 0.007 & 0.015 & 0.018 & 0.032 & 0.009 & 0.011 \\
\hline Log(Income) & 0.047 & 0.048 & 0.029 & 0.042 & 0.055 & 0.050 & 0.046 & 0.033 & 0.046 & 0.048 \\
\hline Male 60-69 & 0.102 & -0.038 & 0.106 & -0.045 & 0.055 & 0.056 & -0.004 & 0.044 & 0.06 & -0.036 \\
\hline Male 70- & 0.025 & -0.184 & -0.225 & -0.164 & -0.174 & -0.160 & -0.155 & -0.368 & -0.178 & -0.195 \\
\hline Female 60-69 & -0.104 & -0.119 & -0.021 & -0.118 & -0.019 & -0.018 & -0.076 & -0.106 & -0.147 & -0.144 \\
\hline Female 70- & -0.165 & -0.290 & -0.450 & -0.238 & -0.234 & -0.200 & -0.193 & -0.495 & -0.333 & -0.286 \\
\hline Self-employed & -0.036 & 0.553 & 0.224 & 0.234 & 0.414 & 0.283 & 0.355 & 0.404 & 0.154 & 0.317 \\
\hline Unemployed & -0.435 & -0.255 & -0.004 & -0.062 & 0.021 & -0.386 & -0.130 & 0.007 & -0.330 & -0.177 \\
\hline Housework & -0.207 & -0.055 & -0.328 & -0.110 & -0.132 & -0.203 & -0.208 & -0.274 & -0.064 & -0.221 \\
\hline Disabled & 0.012 & -0.216 & 0.037 & -0.142 & -0.082 & -0.319 & -0.202 & -0.308 & -0.101 & -0.133 \\
\hline Retired -64 & 0.023 & -0.058 & -0.084 & 0.076 & 0.155 & 0.150 & 0.031 & -0.027 & 0.011 & 0.031 \\
\hline Retired 75- & -0.032 & -0.132 & -0.308 & -0.144 & -0.120 & -0.065 & -0.121 & -0.445 & -0.262 & -0.177 \\
\hline Single & -0.514 & -0.388 & -0.526 & -0.318 & -0.281 & -0.336 & -0.319 & -0.566 & -0.351 & -0.284 \\
\hline Medium education & 0.037 & -0.060 & 0.119 & 0.122 & 0.097 & 0.263 & 0.088 & -0.012 & 0.121 & 0.084 \\
\hline Long education & 0.090 & 0.105 & 0.244 & 0.299 & 0.461 & 0.166 & 0.294 & 0.207 & 0.088 & 0.270 \\
\hline Foreign & 0.001 & -0.004 & -0.006 & -0.005 & 0.037 & NA & -0.081 & 0.002 & -0.022 & -0.023 \\
\hline SAH version 2 & 0.518 & -0.069 & 0.039 & -0.103 & -0.838 & -0.198 & -0.052 & 0.828 & -0.022 & -0.161 \\
\hline
\end{tabular}

Table 6. Concentration indices, Wave 4 


\begin{tabular}{|c|c|c|c|c|c|c|c|c|c|c|}
\hline & Austria & Germany & Sweden & Netherl. & Spain & Italy & France & Denmark & Switzerl. & Belgium \\
\hline Health (pred.) & 0.016 & 0.021 & 0.030 & 0.019 & 0.023 & 0.023 & 0.036 & 0.024 & 0.016 & 0.019 \\
\hline $\mathrm{C}^{*}$ & 0.001 & 0.001 & 0.001 & 0.002 & 0.010 & 0.006 & 0.004 & 0.004 & 0.001 & 0.003 \\
\hline$I^{*}$ & 0.015 & 0.022 & 0.029 & 0.017 & 0.013 & 0.017 & 0.032 & 0.020 & 0.014 & 0.017 \\
\hline$I^{*}$ (\% of pred. health) & 94.29 & 99.37 & 95.17 & 88.50 & 58.14 & 74.96 & 88.90 & 84.59 & 91.73 & 86.09 \\
\hline Log(Income) & 0.045 & 0.048 & 0.038 & 0.042 & 0.066 & 0.058 & 0.045 & 0.032 & 0.045 & 0.056 \\
\hline Male 60-69 & 0.086 & 0.080 & 0.217 & 0.043 & 0.077 & 0.074 & 0.118 & 0.076 & 0.125 & 0.026 \\
\hline Male 70- & 0.005 & 0.062 & -0.114 & -0.185 & -0.096 & -0.052 & -0.133 & -0.363 & -0.122 & -0.078 \\
\hline Female 50-59 & 0.053 & 0.090 & 0.274 & 0.281 & 0.173 & 0.091 & 0.136 & 0.286 & 0.121 & 0.100 \\
\hline Female 60-69 & 0.013 & 0.019 & 0.109 & -0.057 & -0.027 & 0.003 & 0.014 & -0.026 & -0.036 & -0.017 \\
\hline Female 70- & -0.212 & -0.185 & -0.304 & -0.315 & -0.178 & -0.156 & -0.293 & -0.467 & -0.310 & -0.204 \\
\hline Self-employed & 0.086 & 0.190 & 0.506 & 0.463 & 0.289 & 0.363 & 0.338 & 0.373 & 0.201 & 0.218 \\
\hline Unemployed & -0.235 & -0.403 & 0.116 & 0.026 & -0.102 & -0.302 & -0.190 & 0.074 & -0.227 & -0.175 \\
\hline Housework & -0.261 & -0.149 & -0.193 & -0.210 & -0.171 & -0.240 & -0.324 & -0.200 & -0.117 & -0.167 \\
\hline Disabled & -0.216 & -0.257 & -0.111 & -0.223 & 0.002 & -0.295 & -0.312 & -0.152 & -0.302 & -0.200 \\
\hline Retired -64 & 0.025 & 0.123 & -0.037 & 0.185 & 0.110 & 0.203 & 0.086 & -0.004 & 0.058 & 0.074 \\
\hline Retired 65-74 & 0.025 & 0.022 & 0.002 & -0.132 & 0.035 & 0.059 & -0.017 & -0.259 & -0.072 & -0.011 \\
\hline Retired 75- & -0.099 & -0.065 & -0.228 & -0.187 & -0.132 & -0.089 & -0.259 & -0.402 & -0.199 & -0.163 \\
\hline Single & -0.435 & -0.247 & -0.269 & -0.352 & -0.145 & -0.228 & -0.304 & -0.401 & -0.345 & -0.318 \\
\hline Medium education & 0.001 & -0.200 & 0.095 & 0.045 & 0.143 & 0.289 & 0.090 & -0.089 & 0.032 & 0.021 \\
\hline Long education & 0.131 & 0.126 & 0.278 & 0.325 & 0.372 & 0.138 & 0.236 & 0.223 & 0.079 & 0.209 \\
\hline Foreign & -0.048 & -0.009 & -0.017 & -0.157 & -0.318 & -0.164 & -0.024 & -0.165 & -0.201 & -0.099 \\
\hline
\end{tabular}

The concentration index for income has a range between 0.029 for Sweden and 0.055 for Spain (wave 1) while the range is between 0.038 and 0.066 in wave 2 (for the same two countries).

For demographic variables (with males aged 50 - 59 years as reference group) the concentration index decreases from positive to negative values with increasing age in both waves (again, Austria is an outlier in wave 1). Compared to males, females in the age group 50-59 years report better health. For the retired, the concentration indices show a similar picture with decreases from positive to negative values by increasing age (both waves) with few exceptions.

An interesting finding among the income-related distributions of demographic groups is that older males and females tend to be found in the lower income group, in particular in Denmark, followed by Sweden (wave 1) while Denmark is followed by The Netherlands in wave 2. Even younger women 50-59 years can be found in the lower income groups in Denmark closely followed by Germany, Sweden, Spain and Switzerland (wave 1). A similar picture emerges from wave 4, where Denmark is closely followed by Sweden and The Netherlands.

Tables 7 and 8 show, for waves 1 and 4 respectively, contributions from explanatory variables to the concentration indices for predicted health. A positive sign to a variable implies that the concentration index of this variable contributes to increasing inequality. Income contributes positively and to a large extent for 
all countries in both waves, except for Austria. Thus, increasing income inequality contributes to increased income-related inequality in health. In wave 1 , the contribution of income to inequality in health is between $44 \%$ for Switzerland, followed by $42 \%$ and $41 \%$ for Denmark and Sweden. At the lower end of the range of significant results is Spain (12\%) and Belgium (13\%) (wave 1).

In wave 4 , the range has narrowed slightly to between $38 \%$ for Sweden, followed closely by Germany, to $29 \%$ for Denmark and Belgium. Again, Austria is an outlier. In wave 1, the age group 70 years or older men contributes to inequality with Denmark as an exception with a negative contribution implying that income distribution reduces income-related inequality in health. In general, females contribute more in wave 1, still with negative contribution for older Danish females. In wave 4 the contributions from older females have fewer significant results. Interestingly, the contribution has changed to a positive value for men as well as females for Denmark. For older males the contribution decreased for most countries.

Table 7. Contributions, Wave 1

\begin{tabular}{|c|c|c|c|c|c|c|c|c|c|c|}
\hline & Austria & Germany & Sweden & Netherl. & Spain & Italy & France & Denmark & Switzerl. & Belgium \\
\hline Log(Income) & -51.09 & $19.55^{* *}$ & $41.29 * * *$ & $24.25^{* *}$ & 12.03 & $39.63^{* * * *}$ & $35.41^{* * *}$ & $42.06 * * *$ & $44.28^{* * * *}$ & 13.25 \\
\hline Male 60-69 & 9.79 & 1.15 & 0.67 & 0.36 & -0.71 & -0.41 & 0.07 & 1.33 & 0.87 & 0.18 \\
\hline Male 70- & 0.45 & $5.74 * * *$ & $4.69^{*}$ & 2.98 & $9.18^{*}$ & $7.70 * * *$ & $6.92^{* * *}$ & $-8.12^{*}$ & 3.73 & $5.33^{* *}$ \\
\hline Female 50-59 & 3.15 & -0.97 & $-4.50 * * *$ & -0.24 & -3.31 & -1.26 & -0.53 & 1.15 & -0.77 & 0.49 \\
\hline Female 60-69 & -12.03 & 1.82 & -0.15 & -0.81 & 1.34 & 0.60 & $1.27^{*}$ & $-3.45^{* *}$ & 4.98 & $2.90^{*}$ \\
\hline Female 70- & 15.61 & $12.97^{* * * *}$ & $11.89 * *$ & $5.42^{*}$ & $49.75 * * *$ & $18.24 * * *$ & $9.69 * * *$ & $-19.05^{* *}$ & 14.10 & $16.65^{* * * *}$ \\
\hline Self-employed & 0.62 & $3.12^{* * *}$ & 0.50 & 0.47 & -1.10 & 0.90 & -0.70 & -0.45 & 0.51 & -0.65 \\
\hline Unemployed & 13.33 & $3.00 * * *$ & 0.01 & 0.32 & -0.21 & 1.13 & -0.13 & -0.08 & 0.69 & $1.96 * *$ \\
\hline Housework & 18.48 & 1.28 & 0.51 & $5.06^{* *}$ & $13.85^{* *}$ & $11.64 * * *$ & 2.06 & 0.24 & 0.56 & $6.49 * * *$ \\
\hline Disabled & -1.06 & $7.42^{* * *}$ & -0.96 & $19.10^{* * * *}$ & 5.49 & $5.52^{* * *}$ & $6.11^{* * * *}$ & $12.39 * * *$ & 6.39 & $10.02^{* * * *}$ \\
\hline Retired -64 & -6.67 & $1.25^{*}$ & $4.77^{* *}$ & -0.19 & $-5.88 * *$ & $-5.87 * *$ & -0.44 & 1.62 & -0.09 & -0.60 \\
\hline Retired 65-74 & 8.37 & $10.02^{* * * *}$ & $5.24 * * *$ & $10.36^{* * * *}$ & $4.85^{* *}$ & $5.19 * *$ & 2.07 & $18.34 * * *$ & 8.33 & $7.10^{* * * *}$ \\
\hline Retired 75- & 9.58 & $9.07 * * *$ & $16.57^{* * *}$ & $8.49 * * *$ & $10.49 * * *$ & $2.72^{* *}$ & $7.98^{* * *}$ & $36.96 * * *$ & 11.32 & $11.02^{* * *}$ \\
\hline Single & 6.26 & 4.12 & -1.93 & $6.04^{*}$ & -5.79 & 2.14 & -0.54 & -7.96 & -12.16 & 4.60 \\
\hline Medium education & 19.84 & $-6.94^{*}$ & $2.67 * *$ & $4.11^{* * *}$ & $2.16^{* *}$ & $4.91^{* * *}$ & $2.58^{* * * *}$ & -0.69 & 4.32 & $3.76^{* * * *}$ \\
\hline Long education & 20.52 & $7.92^{* * *}$ & $10.59^{* * *}$ & $11.59 * * *$ & $8.73^{*}$ & $2.13^{* * *}$ & $15.31^{* * *}$ & $12.21^{* * * *}$ & $2.18^{* *}$ & $17.29 * * *$ \\
\hline Foreign & -0.01 & 0.01 & 0.03 & 0.06 & 0.05 & NA & $1.36^{* * * *}$ & -0.01 & 0.18 & 0.15 \\
\hline SAH version 2 & 481.03 & -18.95 & 4.70 & -37.70 & -433.09 & -73.13 & -17.01 & 114.04 & -7.80 & -53.12 \\
\hline
\end{tabular}

Note. Significance indicated with ${ }^{*}(10 \%),{ }^{* *}(5 \%)$, and $* * *(1 \%)$

Table 8. Contributions, Wave 8 


\begin{tabular}{|c|c|c|c|c|c|c|c|c|c|c|}
\hline & Austria & Germany & Sweden & Netherl. & Spain & Italy & France & Denmark & Switzerl. & Belgium \\
\hline Log(Income) & $15.58^{*}$ & $36.69^{* * *}$ & $38.18^{* * *}$ & $17.82^{*}$ & 10.38 & 4.22 & $31.70 * * *$ & $28.77^{* * *}$ & $30.60 * * *$ & $28.92^{* * *}$ \\
\hline Male 60-69 & 1.28 & 2.43 & 2.07 & -0.32 & -2.86 & $-2.51^{* *}$ & -0.21 & 0.38 & $-1.89^{*}$ & -0.04 \\
\hline Male 70- & -0.07 & 2.76 & 0.07 & 4.02 & 10.26 & $3.90 * *$ & $2.91^{* * *}$ & $7.50^{*}$ & 2.16 & $2.03^{*}$ \\
\hline Female $50-59$ & $1.59 * * *$ & 3.06 & -1.44 & $-5.34^{*}$ & -4.38 & 0.03 & -0.37 & -0.45 & -0.45 & $-1.48^{*}$ \\
\hline Female 60-69 & 0.92 & 0.92 & 0.83 & 0.51 & 1.48 & -0.15 & -0.03 & -0.50 & 0.39 & 0.20 \\
\hline Female 70- & 1.99 & -8.54 & 3.31 & $12.62^{* *}$ & 37.36 & $23.77^{* * * *}$ & $8.79 * * *$ & 8.47 & $8.05^{* * *}$ & $13.19^{* * *} *$ \\
\hline Self-employed & 0.01 & 0.48 & 0.44 & 0.80 & -0.45 & 1.30 & -0.61 & -1.34 & 0.13 & $1.21^{*}$ \\
\hline Unemployed & $3.16^{* * * *}$ & $5.96^{* * *}$ & -0.58 & -0.11 & 1.03 & $2.04^{* *}$ & 0.34 & -0.89 & $2.24^{*}$ & $2.77^{* * *}$ \\
\hline Housework & $15.09^{* * * *}$ & 0.95 & 0.24 & $6.09^{* * *}$ & 20.32 & $15.89^{* * * *}$ & $2.47^{* * *}$ & -0.21 & 0.79 & $4.56^{* * * *}$ \\
\hline Disabled & $4.55^{* * *}$ & $8.37^{* * *}$ & 2.96 & $16.80 * * *$ & -0.18 & $14.04 * * *$ & $10.65^{* * * *}$ & $8.30 * * *$ & $15.12^{* * * *}$ & $16.30^{* * *}$ \\
\hline Retired -64 & -3.24 & -1.05 & 1.91 & -0.47 & -3.00 & -0.98 & $-1.20 * *$ & 0.05 & -0.10 & $-1.28^{* *}$ \\
\hline Retired 65-74 & $-3.72^{*}$ & -1.60 & -0.19 & $7.84 * * *$ & -2.25 & $-2.33^{*}$ & 0.57 & $5.49 *$ & 1.23 & 0.47 \\
\hline Retired 75- & $10.60^{* * * *}$ & $12.05^{* * *}$ & $25.91^{* * * *}$ & $9.63^{* * *}$ & 13.32 & $8.57 * * *$ & $18.93^{* * *}$ & $17.25^{* * *}$ & $12.51^{* * * *}$ & $10.54^{* * *}$ \\
\hline Single & $29.11^{* * * *}$ & 2.19 & 3.36 & $9.85^{* *}$ & -2.00 & $6.68^{* * * *}$ & 2.86 & $8.15^{*}$ & 1.95 & 3.86 \\
\hline Medium education & 0.04 & $-30.52^{* *}$ & 2.07 & 1.99 & 4.57 & $13.14^{* * * *}$ & $4.01 * * *$ & $-4.33^{*}$ & $4.66^{*}$ & $1.14^{*}$ \\
\hline Long education & $16.88^{* * *}$ & $21.52^{* * *}$ & $10.64^{* * *}$ & $15.71^{* * *}$ & 12.68 & $3.13^{* * * *}$ & $11.91^{* * *}$ & $22.35^{* * *}$ & $4.99^{* * * *}$ & $21.88^{* * *}$ \\
\hline Foreign & $0.78^{*}$ & 0.59 & -0.28 & -0.99 & -0.59 & 3.23 & 0.14 & 0.84 & 1.64 & $-2.52^{*}$ \\
\hline
\end{tabular}

Note. Significance indicated with * $(10 \%), * *(5 \%)$, and ***(1\%)

For most countries, being disabled contributes to income-related inequality in health, but the contribution varies to a large extent which may reflect different access to disability pension in different countries as well as disability compensation. For retired, the contribution increases with increasing age with few exceptions in wave 1. Here, significant results vary from $37 \%$ for those aged $75+$ years in Denmark, followed by Sweden with $17 \%$ to $3 \%$ for Italy and $8 \%$ for France. In wave 4 , the contribution by retired increases by increasing age for all countries. For retired $75+$ years the contribution varies between $26 \%$ for Sweden, followed by $19 \%$ for France and $17 \%$ for Denmark. Finally, groups with tertiary education contribute significantly in most countries.

Table 9 shows the changes from wave 1 to wave 4 in the contributions from the explanatory variables to the concentration index of predicted health by income, calculated as the differences between the contributions of Table 8 and those of Table 7.

Table 9. Development over time: Change in contributions 


\begin{tabular}{|c|c|c|c|c|c|c|c|c|c|c|}
\hline & Austria & Germany & Sweden & Netherl. & Spain & Italy & France & Denmark & Switzerl. & Belgium \\
\hline Log(Income) & 63.67 & 17.14 & -3.11 & -6.41 & -1.65 & $-35.41^{* * *}$ & -3.18 & -13.28 & -13.68 & 15.67 \\
\hline Male 60-69 & -8.51 & 1.27 & 1.39 & -0.68 & -2.14 & -2.10 & -0.28 & -0.95 & $-2.76^{*}$ & -0.22 \\
\hline Male 70- & -0.52 & -2.97 & -4.63 & 1.04 & 1.08 & -3.97 & $-4.01 *$ & $15.62^{* *}$ & -1.56 & -3.29 \\
\hline Female 50-59 & -1.56 & 4.03 & 3.06 & -5.10 & -1.07 & 1.29 & 0.16 & -1.59 & 0.32 & -1.98 \\
\hline Female 60-69 & 12.95 & -0.90 & 0.98 & 1.32 & 0.13 & -0.75 & $-1.29^{*}$ & $2.95^{*}$ & -4.60 & -2.09 \\
\hline Female 70- & -13.62 & $-21.52^{* * *}$ & -8.58 & 7.21 & -12.39 & 5.53 & -0.90 & $27.51^{* * *}$ & -6.04 & -3.46 \\
\hline Self-employed & -0.61 & -2.64 & -0.07 & 0.34 & 0.65 & 0.40 & 0.10 & -0.89 & -0.38 & $1.86^{*}$ \\
\hline Housework & -3.39 & -0.33 & -0.27 & 1.03 & 6.47 & 4.25 & 0.41 & -0.46 & 0.22 & -1.93 \\
\hline Disabled & 5.61 & 0.95 & 3.92 & -2.30 & -5.67 & $8.52^{* *}$ & 5.54 & -4.09 & 8.73 & 6.27 \\
\hline Retired -64 & 3.43 & -2.30 & -2.86 & -0.29 & 2.87 & 4.89* & -0.77 & -1.58 & -0.02 & -0.68 \\
\hline Retired 65-74 & -12.09 & $-11.63 * * *$ & $-5.43^{* *}$ & -2.52 & $-7.09 * *$ & $-7.52^{* * *}$ & -1.50 & $-12.85^{* *}$ & -7.09 & $-6.62^{* * *}$ \\
\hline Retired 75- & 1.01 & 2.98 & 9.34 & 1.13 & 2.83 & $5.85^{* *}$ & $10.95^{* * *}$ & $-19.71^{*}$ & 1.20 & -0.48 \\
\hline Single & 22.85 & -1.98 & 5.29 & 3.81 & 3.79 & 5.54 & 3.40 & $16.12^{*}$ & $14.11^{*}$ & -0.74 \\
\hline Medium education & -19.79 & -23.58 & -0.60 & -2.11 & 2.41 & $8.23^{* *}$ & 1.42 & -3.64 & 0.33 & $-2.62^{* *}$ \\
\hline Foreign & 0.79 & 0.58 & -0.31 & -1.05 & -0.64 & 3.23 & $-1.22^{* *}$ & 0.85 & 1.46 & $-2.67 *$ \\
\hline
\end{tabular}

Note. Significance indicated with ${ }^{*}(10 \%),{ }^{* *}(5 \%)$, and $* * *(1 \%)$

For younger retired below 65, these changes are only marginal. For the middle aged group of age 65-74,

the contribution is reduced for all countries, and significantly so for Germany, Sweden, Spain, Italy, Denmark and Belgium. However, given that these changes are composed by changes in regression coefficients (expressing changes in relative health status) as well as changes in concentration indices (expressing change in relative economic status) for the age group, the patterns underlying the reductions varies. Thus, for some countries, an improvement in health is predominant, while for other an improvement in economic status is. By comparing tables 3 and 4, Denmark, Switzerland, Italy and Germany are seen to be predominant representatives of the former group, as their regression coefficient is reduced, while comparison of tables 5 and 6 shows that most countries experience improves in the relative economic status of the age group.

For the elder groups of retired 75 years and above, the changes in contributions are moderate and only significant for Italy, France and partly Denmark. However, while the contribution is reduced for Denmark, it appears to have increased for Italy and France. For the last two countries, the regression coefficients of the age group increases in magnitude, thus reflecting a worsening of the relative health status of the group, while health status is improved for Denmark Table 3 and 4). Furthermore, the concentration indices are increased for Italy and France, thus reflecting a worsening of the relative economic status of the group, while it is approximately unchanged for Denmark (Table 5 and 6). 
Turning to the specific hypotheses, the first question is whether the relative high concentration index that was found in Denmark in earlier studies based on samples of the whole adult population (Christiansen, 1997; van Doorslaer and Koolman, 2004), also can be found in SHARE data, based on a population of 50 years or more, and based on later surveys. Table 5 clearly demonstrates that this is the case for wave 1. When comparing countries, it appears from wave 1 (2004-2006) that the concentration index for health is the highest in Denmark (0.025). However, turning to wave 4 (2011-2012) the size of the Danish concentration index is almost unchanged (0.024), while the indices are substantially higher for France (0.036) and Sweden (0.030).

Adjustment for age and gender provides a measure of what may be labelled "avoidable" inequalities (I*). It appears that adjustment for age and gender increases the health concentration index for Denmark in wave 1 unlike what is the case for other countries. However, in wave 4, the age- and gender adjustment decreases the avoidable inequality to 0.020 which is in between the extreme values. In conclusion, while the unadjusted concentration indices are relatively high for Denmark in both waves, the avoidable health inequality has decreased between the two waves, and Denmark is no longer a country with exceptional income-related inequality in "avoidable" health.

Hypothesis 1 is related to pensioners' share of the total sample ( $\bar{x}_{k}=$ pensioners' share $)$ seems confirmed for wave 1, unlike wave 4. While pensioners in Denmark made up $55.1 \%$ of the sample in wave 1 , compared to $44.7 \%$ for the other countries, the corresponding shares are 47.1 and $54.5 \%$ (table 1 and 2, unweighted averages). In conclusion, the data only supports hypothesis 1 for wave 1 , but not for wave 4

Hypothesis $2 \mathrm{a}$ is about the relative health of the retired in Denmark, compared to the other countries. The $\beta$-coefficients in table 3 and 4 show that in general retirement is associated with lower health to a greater extent in Denmark compared to other countries and thus the hypothesis is supported for wave 1. In wave 2 , this is however not the case.

Hypothesis $2 \mathrm{~b}$ is about this association of health status and age of retired in Denmark. It appears that the association is of about equal size for the younger pensioners and the elderly in the both waves. Thus, the 
hypothesis of decreasing health by increasing age of pensioners could not be supported. Still, the size of the associations has decreased for all age groups from wave 1 to 4 , implying relatively better health of pensioners over time.

Hypothesis $3 a$ is about the relative income of Danish pensioners, compared to pensioners in other countries. This works through $C_{k}$ (table 5 and 6). In neither of the two waves were the coefficients outside the range for other countries. The hypothesis could not be supported.

Hypothesis $3 \mathrm{~b}$ is about the relative income (relative to income of working individuals in the sample). It appears that over time the income inequality has been reduced for the two youngest age groups among pensioners, and thus the hypothesis is supported.

Hypothesis 4 is about the contribution to income-related health inequality be the middle age group among pensioners. It appears from table 9 that the hypothesis can be supported. However, the contribution from the oldest age group has decreased as well.

\section{Discussion}

While the index of income-related inequality in health merely provides a summary measure, it is possible by means of the decomposition to get a deeper insight into measured inequality. Compared to the analyses by van Doorslaer and Koolman (2004) we have further divided retired into three age groups rather than two which allows a more precise analysis of the contributions to inequality by retired. The reference group for retired is wage earners, and our focus is on differences between wage earners and retired in Denmark, compared to other countries in the study. In general the contribution from retired to inequality in incomerelated health varies very much between countries and between age groups. While this is related to three determining factors as well as unobserved residual inequality, it is difficult to find a coherent pattern across countries.

In contrast to the suggestions by van Doorslaer and Koolman (2004) however, we find that it is especially the oldest among the retired who contribute to income-related inequality in health in both Denmark and in 
most other EU countries in both waves. If the retired in Denmark over 75 years had the same health status and income as the rest of the sample, $37 \%$ of the measured inequality would disappear in wave 1 and $17 \%$ in wave 2. This comes on the top of the effect of age per se which has also a substantial contribution, in particular for Denmark in wave 1.

While the findings support those from an OECD survey (OECD, 2013) that disposable income of persons less than 65 years are relatively low in Denmark, they do not imply that older people in Denmark are in a worse position in an absolute sense, compared to inhabitants in other countries; what it means is that their income relative to the income by younger generations is lower. Moreover, disposable income does not account for rebates and services which pensioners receives from the public sector, or for a possible dissaving of their fortune.

The different contribution from retired to the inequality index across countries is to a large extent associated with different relative income levels by retired. This may be ascribed to different pension schemes as well as possibilities or habits in various countries in having supplementary income through jobs for retired.

Turning to Denmark as an outlier in wave 1 , we found a relatively high concentration index for predicted health - a finding that corresponds with earlier findings (Christiansen, 1997, van Doorslaer and Koolman, 2004). This was intuitively unexpected when comparing the distribution of income across countries. From our analysis it appears that in particular the older retired contribute to the inequality health of the Danes, and income distribution in itself also contributes, although to a smaller extent. In contrast, van Doorslaer and Koolman (2004) found in their analysis that income distribution contributed with almost nothing while retirement contributed with $91 \%$, and they concluded that the findings for Denmark should be attributed to a disadvantaged position of the early retired. Their reasoning departed from the observation that the contributions should be interpreted as partial effects, i.e. after having controlled for demographics and income. Thus, the retired report worse health than others of the same age and income, and they concluded that it therefore mainly reflect the disadvantaged position of the early retired (p. 622). In contrast, we found that the results are attributable to the oldest among the retired. The reason for this high 
contribution to health inequality by older retired Danes may be a combination of a skew income distribution in disfavour of the older retired, and older retired being in less than average health of the population.

Adjustment for demographics is part of the explanation for a high concentration index in wave 1 , but less in wave 4. Denmark is the only country where relative health status has improved between the two waves for the medium age group of 65-74 year old retired as well as the elder retired of 75 years and above. Furthermore, the relative economic status of the retired age group 65-74 has improved, while it is almost unchanged for the retired elder group of 75 and above. Thus, Denmark is the only country experiencing a reduction in the relative contributions to health inequality for the two age groups simultaneously. Two major explanations are relevant. First, retirement savings etc. became increasingly widespread in the age group 65-74 between the two waves, thus improving the relative economic status of the group. Second, relative health status has improved in both age groups, presumably as a result of an increased focus on public health initiatives, of which a part has been directed to the elder while another part may be due to earlier initiatives.

For Denmark, the spread in pension savings and benefits cannot yet be observed in the older age group of 75 and above, as expressed by the unchanged concentration index for this group. However, it is expected to be in close future, thus improving the economic status of the group. In connection with a continued improvement in relative health status for the group, the contribution of the group to health inequality is expected to be further reduced.

As to international comparisons of self-reported health there has been documented to be large variations across countries that to a certain extent may be due to differences in reporting style rather than health (Jürges, 2007). Accordingly, e.g. Danes tend to overrate their health (compared to the average) while Germans and people in Southern Europe tend to underrate. Whether this seemingly pattern affects the "true" distribution of health has still to be explored. 
The policy implications for Denmark seems to be that, as far as the retired are concerned, much of the contribution to inequality in income-related health stems from income inequality and to a less extent from health inequality. This will probably disappear with a still increasing use of labour market pension schemes in addition to the public financed universal pension scheme which will make the older age group better off economically, compared to today. Still, as pointed out by the Danish Economic Council (Det $\varnothing$ konomiske Råd, 2008), there exists a marginal group without this supplementary pension scheme.

Given the careful effort behind the collection of SHARE date, including pre-test sampling, face-to-face interviews etc., the validity of the data as well as of the results presented in this study are considered to be extremely high.

Calculation of the Concentration index and decomposition of health (Wagstaff et al., 2003) has been subject to discussion and suggestions for corrections. A review thereof can be found in van Doorslaer and van Ourti (2011). We address some of these in the following.

Linearity of the relationship between the explanatory variables and health is an assumption, which is for discussion. For the case of age and gender, this has been resolved by using age categories and interactions between these and gender as suggested by van Doorslaer and Koolman (2004). For some variables, which are coded as binary indicators, the matter is not relevant. However, it is an open discussion as to whether income should enter in linear or some non-linear form (van Doorslaer and Koolman, 2004; van Doorslaer and van Ourti, 2011).

Furthermore, exogeneity of the explanatory variables may be an issue. In particular, the relationship between income and health has been much discussed, given that income may as well be formed by health. The issue was discussed recently by Heckley et al. (2016), who suggested a new methodology which explicitly addressed the endogeneity. Another suggestion has been to use education instead of income as measure of socioeconomic status, given that education is formed relatively early in life and therefore to a less extent affected by present health (Arendt and Lauridsen, 2008). 
Another point regards the assumption that the determinants of health do not affect the rank. However, this conflicts with $\mathrm{Cl}$ being defined from the covariance between health and income rank. Originally, the $\mathrm{Cl}$ was suggested as a measure of univariate income distribution and later used by Wagstaff et al. (2003) and later authors to describe the bivariate distribution between health and income. Erreygers and Kessels (2013) discussed this problem in details suggested different bivariate approaches. Later, Kessels and Erreygers (2014) and Erreygers and Kessels (2015) considered the simultaneity between income and health by introducing a Structural Equation Model (SEM) approach. Yet another approach has been to discard the $\mathrm{Cl}$ approach and use a multivariate structural model for "unfair health", based on which the inequality in health can be summarized in different ways (Fleurbaey and Schokkaert, 2009).

The present paper can be seen as a follow-up on the results for Denmark that was shown in van Doorslaer and Koolman (2004) and therefore we have decided to use the same methods, including the original concentration index. We are aware of suggested correction to the concentration index by Wagstaff (2005) and Errygers (2009) but we have kept the original method to facilitate comparisons of previous results with the results from the present paper. Wagstaff showed that that the upper and lower bounds of a binary variable whose inequality is investigated depend on the mean of this variable, while Errygers showed that this is the case for any variable with bounds. Thus, when a health variable has bounds, the concentration index will depend on the mean, and comparisons between populations with different health means therefore become problematic.

In their decomposition Wagstaff et al. (2003) showed that the Concentration index can be decomposed into a deterministic and a residual component defined by $2 *$ the covariance between the error term and the rank variable (socio-economic status or income). As pointed out by Kessels abd Erreygers (2014, p.5-6) the introduction of a socioeconomic variable in the regression of health creates a problem because the covariate between health and the error term will be zero or close to zero, implying that all or most of the variation of the Concentration index has been explained. They claim that this is an artefact due to the OLSbased approach. 
As stated above, we are also aware of recent developments of decomposition methods considering that socio-economic inequality is bivariate by nature and measuring the correlation between the two variables, health and socio-economic status (Erreygers and Kessels, 2013; Kessels and Erreygers, 2014; Erreygers and Kessels, 2015). To these, Heckley et al. (2016) added a Recentered Influence Function (RIF) regression approach, where a two-dimensional decomposition of determinants of health as well as of determinants of the socio-economic variable (income in the present paper), together with a feed-back between these two, was suggested. However, we have kept the original decomposition method of health alone for the same reason as above.

The choice of concentration index involves a value judgement as discussed by e.g. Allanson and Patrie (2013) and Kjellson et al. (2015). Thus, a choice has to be made between absolute and relative measures (where the calculations in the present paper are based on a relative index), and between measures of health or ill-health in case the index has both a lower and an upper bound (where self-assessed health with a lower and upper bound has been used in the present paper). As shown by van Doorslaer and Koolman (2000) and Clarke et al. (2002), the choice of index can influence the ranking. 


\section{Literature}

Allanson P, Paetrie D. (2013). On the choice of inequality measure for the longitudinal analysis of incomerelated health inequalities. Health Economics 22:353-365.

Arendt J, Lauridsen J. (2008). Do risk factors explain more of the social gradient in self-reported health when adjusting for baseline health? European Journal of Public Health 18: 131-137.

Börsch-Supan A, Brandt M, Hunkler C, Kneip T, Korbmacher J,Malter F, Schaan B, Stuck S and Zuber S on behalf of the SHARE Central Coordination Team (2013). Data Resource Profile: The Survey of Health,Ageing and Retirement in Europe (SHARE). International Journal of Epidemiology 42:992-1001.

Christiansen, T. (1997). Distribution of Self-Assed Health by Income. Empirical results from Denmark. Odense University CHS Working Paper 1997:9.

Christiansen T, Lauridsen J, Häkkinen U. (2009) Determinants of inequalities in health with focus on retired with particular regard to retired Danes. Health Economics Papers 2009 :4, University of Southern Denmark. http://static.sdu.dk/mediafiles//1/A/B/\%7B1ABEBBC8-DD24-400F-A46B-A076E4A72F27\%7D20094.pdf

Clarke PM, Gerdtham U-G, Johannesson M, Bingefors K, Smith L. (2002). On the measurement of relative and absolute incomwe-related inequality. Social Science \& Medicine 55: 1923-1928.

Deaton A. (1997). The Analysis of Household Surveys: A Microeconometric Approach to Development Policy. Baltimore: John Hopkins University Press.

Det Økonomiske Råd (2008) [Danish Economic Council]. Danish Economy, Spring 2008. Savings (in Danish, with English summary). Copenhagen.

Efron B, Tibshirani RJ (1993). An Introduction to the Bootstrap. Chapman \& Hall : London.

Errygers G. (2009). Correcting the concentration index. Journal of Health Economics 28: 504-513. 
Erreygers G, Kessels R. (2013). Regression-based decompositions of rank-dependent indicators of socioeconomic inequality of health. Working Papers 2013007, University of Antwerp, Faculty of Applied Economics.

Erreygers G, Kessels R. (2015). Socioeconomic status and health: A new approach to the measurement of bivariate inequality. Working Papers 2015-017, University of Antwerp, Faculty of Applied Economics.

Eurostat (1999). European Community Household Panel (ECHP),

http://ec.europa.eu/eurostat/web/microdata/european-community-household-panel

Fleurbaey M, Schokkaert E. (2009). Unfair inequalities in health and health care. Journal of Health Economics 28: 73-90.

Heckley G, Gerdtham U-G, Kjellson G. (2016). A general method for decomposing the causes of socioeconomic inequality in health. Journal of Health Economics 48: 89-106.

Jones A. (2000). Health Econometrics. In: Handbook of Health Economics. Culyer AJ, Newhouse JP (eds). Elsevier : Amsterdam.

Jürges H. (2007). True health versus response styles: Exploring cross country differences in self-reported health. Health Economics 16: 163-178.

Kakwani N, Wagstaff A, van Doorslaer E. (1997). Socioeconomic inequalities in health: Measurement, computation, and statistical inference. Journal of Econometrics 77: 87-103.

Kessels R, Errygers G (2014). A unified structural equation modeling approach for decomposition of rankdependent indicators of socioeconomic inequality of health. Research Paper 2014-013, Department of Economics, University of Antwerp.

Kjellson G, Gerdtham U-G, Petrie D. (2015). Lies, damned lies, and health inequality measurements. Understanding the value judgement. Epidemiology 26: 673-680. 
OECD (2013). Pensions at a Glance 2013. http://www.oecd-ilibrary.org/finance-and-investment/pensionsat-a-glance-2013/relative-incomes-of-the-over-65s-late-2000s pension glance-2013-graph13-en

SHARE-project (2015). Research Data Center and Data Access. http://www.share-project.org/data-accessdocumentation/research-data-center-data-access.html

van Doorslaer E, Jones A (2003). The determinants of inequalities in self-reported health : validation of a new approach to measurement. Journal of Health Economics 22: 61-87.

Van Doorslaer E, Koolman X (2000). Income-related inequalities in health: some evidence from European Community Household Panel. Equity II Project Working Paper \#1, Erasmus University, Rotterdam.

van Doorslaer E, Koolman X (2004). Explaining the differences in income-related health inequalities across European countries. Health Economics 13: 609-628.

Van Dorslaer E, van Ourti T (2011). Measuring inequality and inequity in health care. Ch 5 in: Glied S and Smith PC (eds.), The Oxford Handbook of Health Economics, Oxford: Oxford University Press: 837-869

van Doorslaer E, Wagstaff A, Bleichrodt H (1997). Income-related inequalities in health: Some international comparisons. Journal of Health Economics 16: 93-112.

van Doorslaer E, Wagstaff A, Rutten F, eds. (1993). Equity in the finance and delivery of health care. Oxford University Press.

Wagstaff A. (2005). The bounds of the Concentration Index when the variable of interest is binary, with an application to immunization inequality. Health Economics 14: 649-653.

Wagstaff A, van Doorslaer E, Watanabe N (2003). On decomposing the causes of health sector inequalities with an application to malnutrition in Vietnam. Journal of Econometrics 112: 207-223. 
